\title{
CAPÍTULO II \\ EFECTOS DEL CAMBIO CLIMÁTICO SOBRE LAS TASAS DE TRANSPORTE DE SEDIMENTOS EN GRANDES RÍOS: UNA REVISIÓN
}

\section{José Luis Lugo}

Magíster en Ingeniería Ambiental, Investigador de los grupos de investigación: Grupo Udecino de Investigación Ambiental Universidad de Cundinamarca, y Suelo, Ambiente y Sociedad Universidad del Magdalena. Emails: jllugo@ucundinamarca.edu.co

\section{Elkyn Rafael Lugo}

Magister en Economía, Docente Investigador del grupo Desoge de la Corporación Universitaria Minuto de Dios Uniminuto. e-mail: elkyn.lugo@uniminuto.edu.co y elkynlugo@gmail.com.

\section{Javier Burgos Vergara}

Doctor, Docente Investigador de la Universidad del Sinú. E-mail: javierburgos@ unisinu.edu.co

\section{David Andrés Crespo Tovar}

Magister en Ingeniería Civil, Profesor Tiempo Completo de la Universidad Cooperativa de Colombia (UCC) sede Santa Marta. E-mail: david.crespo@campusucc.edu.co

\section{Jonathan Castro Mercado}

Especialista en Estadística Aplicada; Docente Catedrático en la Corporación Universitaria Minuto de Dios (Uniminuto) y en la Institución Universitaria de Educación Superior (ITSA). E- mail: uajcastromercado@hotmail.com y jcastromerc@gmail.com

\section{Resumen}

La respuesta de la descarga de agua y sedimentos de río al cambio climático y a la actividad humana es un tema que ha despertado mucho interés en todo el mundo; sin embargo, la mayoría de los estudios se han enfocado principalmente en las actividades humanas, especialmente en la construcción de presas, que se ha identificado como la causa principal de la tendencia decreciente en las cargas de sedimentos de los ríos; adicionalmente analizar los factores que afectan el transporte de sedimentos (cambio climático y diversas actividades humanas) en grandes ríos es difícil y se requiere de análisis complejos para su determinación desde un enfoque holístico. Es por ello que el presente trabajo tuvo como objetivo revisar la literatura científica sobre las implicaciones que tiene el cambio climático en las tasas de aporte de sedimentos en grandes ríos, así como su relación con otros factores que también afectan el transporte de sedimentos. Esta investigación permitió concluir que es importante analizar el cambio climático de manera simultánea e integrada con el desarrollo de actividades humanas en cuencas hidrográficas (como por ejemplo: agricultura, construcción de presas, turismo, embalses, entre otras), ya que se han presentado casos de estudio en los cuales la afectación de las tasas de transporte de sedimentos ha sido más influenciada por el uso de suelo (como por ejemplo: la construcción de presas), que 
por el cambio climático, que aunque este último es evidente, muchas veces su análisis individual no representa bien su grado de incidencia sobre el transporte de sedimentos, ya que al no analizar las actividades antropogénicas, en especial en cuencas muy ocupadas y/o gestionadas con programas de conservación de agua y suelo, se correría el riesgo de tener errores muy altos en las estimaciones realizadas.

Palabras clave: Tasas de transporte de sedimento, cambio climático, grandes ríos.

\title{
EFFECTS OF CLIMATE CHANGE ON SEDIMENT TRANSPORT RATES IN LARGE RIVERS: A REVIEW
}

\begin{abstract}
The water and sediments discharge response to climate change and human activity is a topic of great interest throughout the world. However, the majority of studies have focused mainly on human activities, especially on dams building, which have been identified as the main cause of the decreasing of load sediments in rivers. Complex analyses are required to a holistic comprehension of factors that affect the transport of sediments in large rivers. The present work aimed to review the scientific literature on the implications of climate change and sediment supply rates in large rivers. As well as the relationship between changes in sediments movements and other factors that may affect. The present paper allowed to highlight the importance of climate change and human development activities in watersheds (such as agriculture, dam construction, tourism, reservoirs, among others) analyses integrated. Cases of study have shown sediment transport rates has been more influenced by the use of land (as for example: the construction of dams), than by climate change. Individual analysis might not represent well the degree of incidence on sediment transport, since leaving behind anthropogenic activities, especially in very busy basins and / or managed with water and soil conservation programs, would have errors in the estimations done.
\end{abstract}

Keywords: Sediment transport rates, climate change, great rivers.

\section{Capítulo resultado de un proyecto de investigación culminado.}

\section{Introducción}

El cambio climático se ha observado en las últimas décadas y puede proyectarse al futuro (IPCC, 2007). Estos cambios principalmente en la precipitación y temperatura y su efecto en el transporte de sedimentos en cuerpos de agua (como por ejemplo: en ríos) es una preocupación actual en la investigación (Mullan, Favis-Mortlock \& Fealy, 2012; Bussi, Francés, Horel, López \& 2014) ya que el aumento de la temperatura global afecta la extensión, frecuencia y magnitud de la erosión del suelo y la redistribución del suelo (Pruski \& Nearing, 2002), que pueden alterar la dinámica sedimentaria de una cuenca hidrográfica. 
Así mismo, las actividades humanas afectan en la movilización y transporte de sedimentos (Wei, He, \& Bao, 2011); como por ejemplo: el desarrollo agrícola, preparación del suelo para cultivos (labores agrícolas), extracción de minerales, urbanización y desarrollo infraestructural, construcción de presas y de embalses, y programas de conservación de suelos y control de sedimentos (Shrestha et al., 2013; Quynh, Garnier, Gilles, Sylvain \& Minh, 2007).

La alteración de la dinámica sedimentaria de los ríos podría ocasionar problemas ambientales y económicos graves. En lo ambiental, las altas concentraciones y cargas de sedimentos en ríos reducen la cantidad de luz solar disponible para las plantas, produciendo pérdida de especies vegetales; puede provocar sofocación de perifiton y macrofitas; se podría reducir la capacidad respiratoria de los peces (Waters, 1995; Milhous, 1996) citado en (Beltaos, 2016); los sedimentos suministrados por la erosión de las zonas agrícolas son responsables del transporte de agroquímicos, entre los que destacan los plaguicidas a los ríos (Yao \& Xu, 2013); la disminución del transporte de sedimentos al océano afecta a los ciclos biogeoquímicos, y por ende a la salud de las especies en zonas costeras (Potemkina \& Potemkin, 2015; Wang et al., 2011). En lo económico, se podría afectar la operatividad de los canales de navegación debido a la agradación causada por el incremento en las tasas de aporte de sedimento, lo que representaría altas inversiones en obras de dragado del lecho de determinado río (Re, Kazimierski \& Menéndez, 2014; Kazimierski, Re \& Menéndez, 2011). De ahí la importancia de evaluar el efecto de los cambios en el clima y en el uso del suelo (actividades humanas) sobre el transporte de sedimentos en los ríos, para permitir que los gestores de recursos hídricos tomen decisiones adecuadas de planificación y control del manejo de cuencas hidrográficas (Shrestha, Maskey, Babel, Griensven, Uhlenbrook, 2016).

El estudio de los factores que afectan el transporte de sedimentos en grandes ríos es muy difícil (Gómez, Cui, Kettner, Peacock \& Syvitski, 2009); pues intervienen diferentes actividades humanas que alteran la dinámica sedimentaria en estos sistemas fluviales que van cambiando en el tiempo como también lo hace el cambio climático, que de hecho, determinar su tendencia es compleja, dado que su comportamiento no es lineal, y por ende presenta incertidumbre en su estimación. El cambio climático y las acciones humanas sobre la cuenca pueden tener efectos sinérgicos o antagónicos, por lo que deben analizarse simultáneamente (Bussi, Dadson, Prudhomme \& Whitehead, 2016).

La respuesta de la descarga de agua y sedimentos del río al cambio climático y a la actividad humana es un tema que ha despertado mucho interés en todo el mundo; sin embargo, la mayoría de los estudios se han enfocado principalmente en las actividades humanas, especialmente en la construcción de presas, que se ha identificado como la causa principal de la tendencia decreciente en las cargas de sedimentos de los ríos (Zhao et al., 2015). Dada la relevancia e importancia de esta temática, el presente trabajo tiene como objetivo revisar la literatura científica sobre las implicaciones que tiene el cambio climático en las tasas de sedimentos en grandes ríos, así como su relación con otros factores que también afectan el transporte de sedimentos. Esta revisión permitirá al lector tener una visión integrada desde el punto de vista de la ingeniería y la gestión para que esta problemática sea enfocada por los tomadores de decisiones del recurso hídrico en el contexto global. 


\section{Fundamentación teórica}

\section{Clima, tiempo meteorológico y cambio climático}

El tiempo meteorológico hace referencia al estado de la atmósfera en un determinado día, semana o mes; se caracteriza por la humedad, la temperatura, la presión, las precipitaciones y la nubosidad en un determinado lugar y momento (PNUMA, 2005). El clima es el conjunto de fenómenos meteorológicos que caracterizan el estado medio de la atmósfera en una región del planeta, como temperaturas medias, precipitaciones medias, vientos dominantes, etc. (PNUMA, 2005); por lo tanto, es el promedio del tiempo meteorológico en un largo plazo (Fuentes, 2000). Por su parte, el cambio climático es el cambio del clima a través del tiempo como resultado de la variabilidad natural o de las actividades humanas, lo que desencadena la presencia de estados de tiempo extremos, tanto húmedos (altas precipitaciones) como secos (ausencia de precipitación y altas temperaturas), condiciones que afectan a los ecosistemas y a la vida del planeta (SEMARNAT, 2009).

\section{Transporte de sedimentos en ríos y su relación con el cambio climático}

Los sedimentos en los sistemas acuáticos, incluyendo ríos, hacen referencia a partículas gruesas o finas que tienden a depositarse en el fondo de estos sistemas naturales. Estas partículas son importantes porque aportan nutrimiento a los seres vivos que allí habitan (Wang et al., 2011) y son fundamentales para el ingreso de material en los sistemas costeros, como es el caso de la formación de línea de costas (Liu, Sui, He \& Hirshfield, 2013).

El transporte de sedimentos en ríos hace referencia al movimiento de las partículas en estos cuerpos de agua, lo cual se hace a través de estimaciones matemáticas que resultan complejas. El arrastre o transporte de fondo se refiere a las partículas del fondo (partículas de mayor tamaño) que se mueven o deslizan en la sección transversal del río; mientras que las de suspensión son las partículas finas que se encuentran distribuidas en prácticamente toda la sección transversal del río y que son transportadas por la corriente de agua (Rocha, 1998)

En los ríos, el movimiento de sedimento depende de condiciones hidráulicas e hidrológicas que determinan el flujo de agua, y por ende el de las partículas en estos ecosistemas. Sin embargo, el cambio climático al alterar las condiciones hidráulicas como producto del aumento o disminución extremos principalmente de precipitaciones, que incide en los flujos de agua, que tienen capacidad de transporte de sedimento (Pruski \& Nearing, 2002). Al ser estos fundamentales en los ecosistemas acuáticos, es importante analizar el efecto del cambio climático sobre las tasas de transporte de sedimento en ríos, así como en otros sistemas naturales, lo cual se ha estudiado poco y se ha despertado el interés actual en el campo científico (Mullan et al., 2012; Bussi et al., 2014), como se mencionó anteriormente.

\section{Metodología}

Se realizó una búsqueda bibliográfica en las bases de datos Science Direct, Redalyc y el buscador académico "Google Académico", usando combinaciones de las siguientes 
palabras claves: "Transporte de sedimentos en ríos", "efecto del cambio climático sobre el transporte de sedimentos en grandes ríos" y "cambio climático y transporte de sedimentos en ríos". En inglés: "sediment transport in rivers", "effect of climate change on the transport of sediments in large rivers", "Climate change and transport of sediments in rivers".

Los criterios para la selección de los artículos científicos fueron los siguientes: (1) el estudio analizó el efecto del cambio climático sobre el transporte de sedimento en ríos, (2) se estimó la producción de sedimento en periodos largos de tiempo usando métodos pertinentes para tal fin; (3) presentó interés en el impacto de la variación en el transporte de sedimento sobre ecosistemas acuáticos, y (4) se realizaron análisis del impacto del cambio de uso de suelo sobre el transporte de sedimento en grandes ríos. Con base en la revisión de los artículos seleccionados, se procedió a su comparación y extracción de ideas más importantes, con el objeto de analizar la problemática asociada con los efectos del cambio climático y el uso de suelo sobre las tasas de transporte de sedimento en grandes ríos como herramienta para la toma de decisiones en la planificación y gestión del recurso hídrico. Esta investigación descriptiva surge por la necesidad de analizar los efectos del cambio climático sobre grandes ríos y su interacción con las actividades humanas, ya que se ha venido estudiando el impacto generado por actividades humanas (proyectos de ingeniería de aprovechamiento de recursos hídricos, como la construcción de presas), como se mencionó anteriormente, pero el efecto del cambio climático ha cobrado relevancia últimamente y hay poca información sobre el análisis en conjunto con las actividades humanas (Bussi et al., 2016), por lo que esta revisión pretende describir cómo afecta el cambio climático a las tasas de transporte de sedimento en grandes ríos, así como los análisis y las técnicas que se han realizado para abordar estas problemáticas asociadas al recurso hídrico.

\section{Resultados y discusión}

\section{Influencia del cambio climático en el transporte de sedimentos}

El clima juega un papel importante en el transporte de sedimentos y su análisis es importante para entender el comportamiento del flujo de sedimentos en grandes ríos y su afectación sobre el entorno natural y social como se mencionó en la sección anterior. En un estudio realizado por Beltaos \& Burrell (2016) se evaluó el transporte de sedimentos en el río Saint John en Canadá mediante medición directa de los sedimentos en suspensión durante el derretimiento de hielo; y se encontró una relación entre la influencia climática y la carga de sedimentos, a medida que la temperatura aumentó también lo hizo la carga de sedimentos en el río, proyectándose tendencias crecientes con el calentamiento global.

Por otro lado, Muñoz \& Castillo (2015) determinaron la respuesta del caudal y la carga de sedimentos atribuidos al fenómeno de la Oscilación del Sur-el Niño (ENSO) y la niña, en el mayor sistema fluvial de México: la cuenca de los ríos Usumacinta y Grijalva. Para ello analizaron datos de descarga de agua y carga de sedimentos durante 56 años, y los resultados mostraron que las magnitudes de estas dos variables fueron mayores en el periodo de La Niña. Conclusiones similares se obtuvieron en el estudio realizado por Bogen (2009), en el que se evaluaron las cargas de sedimentos en varios ríos noruegos durante eventos extremos de precipitación y se determinó que la tasa de sedimento incrementó durante 
las inundaciones que ocurrieron después de un largo periodo lluvioso. Khan, Daityari \& Chakrapani (2016) examinaron el patrón de la variación espacio-temporal en el flujo de agua y de sedimentos del río Ramganga (India) mediante análisis de datos diarios de caudal y concentración de sedimentos durante 10 años, y encontraron asociación entre la reducción de descarga y transporte de sedimento durante El Niño.

Otro enfoque existente para evaluar el impacto del cambio climático en el transporte de sedimentos es el análisis paleoambiental (Scherler, Bookhagen, Wulf, Preusser \& Strecker, 2015), los cuales pueden relacionarse con los cambios climáticos y con la evolución de la población humana (Bogaart \& Balen, 2000; Mei'e, 2015). Marrero et al. (2014) realizaron un análisis paleoambiental (durante los últimos 100 años) a partir del isótopo ${ }^{210} \mathrm{~Pb}$ para la datación de dos testigos de sedimentos (GeoB 13813-4 y BAR1) relacionándolos con forzantes climáticas como el fenómeno de El Niño, La Niña, Oscilación Decadal Pacifica (ODP) y las anomalías hidrológicas de los ríos Paraná y Uruguay. En este estudio se encontró que la tasa de sedimentación estuvo influenciada por la variabilidad hidroclimática del siglo XX. Owens \& Walling (2002) examinaron núcleos de sedimentos extraídos de las llanuras inundables de tres sitios de la cuenca de Tweed (Escocia) para reconstruir la tasa de sedimentación en la llanura de inundación en los últimos 100 años, y concluyeron que el cambio climático por sí solo no pudo explicar la tendencia decreciente de las tasas de sedimento, pero estos cambios parecieron tener mayor relación con el cambio de uso de suelo durante el lapso de estudio.

No obstante, debido a la complejidad y a la no linealidad, la forma más habitual para evaluar los efectos del cambio climático en los ciclos de agua y sedimento es mediante la modelación matemática (Francés \& Bussi, 2015). Esta herramienta es útil en ingeniería, porque permite establecer y determinar escenarios para la toma de decisiones. Asselman, Middelkoop \& Dijk (2004) establecieron una serie de modelos de Sistemas de Información Geográfica (SIG) para simular la producción y el transporte de la carga de lavado en el curso inferior del río Rin (Europa), en el que encontraron que, aunque las descargas en el río se esperan muy altas con mayor frecuencia, la sedimentación en las llanuras de inundación tiende a disminuir.

Francés \& Bussi (2015) analizaron el impacto del cambio climático en el ciclo de sedimentos de la cuenca del río Ésera (España) mediante un modelo TETIS de la Universitat Politècnica de València, seleccionando escenarios futuros de emisión de $\mathrm{CO}_{2}$ definidos por Special Report on Emissions Scenarios (SRES) encontraron que en el escenario más pesimista de cambio climático, el transporte de sedimento tendió a disminuir; mientras que en el más conservacionista tendió a aumentar, lo que es importante evaluar cuando se analizan supuestos de variación de la tendencia del cambio climático. Darby, Dunn, Nicholls, Rahman \& Riddy (2015) mediante la aplicación del modelo HydroTrend concluyeron que se prevén incrementos de suministro de sedimentos con respecto al cambio climático en el mega-delta Ganges-Brahmaputra (Asia).

Shrestha et al. (2013) evaluaron el impacto de un posible clima futuro sobre el rendimiento sedimentario en la cuenca del río Nam Ou (Lao RDP), usando el modelo SWAT para evaluar los cambios que ocasionará el cambio climático sobre el flujo de sedimento. En este trabajo se encontró que se esperan descargas más altas y flujos de sedimentos durante la estación húmeda, aunque los cambios porcentuales serían mayores durante los 
meses secos. Por otro lado, Verhaar, Biron, Ferguson \& Hoey (2008) adaptaron un modelo morfodinámico unidireccional desarrollado para lechos de grava (SEDROUT), el cual fue validado para un periodo de 100 años en el río Saint Lawrence. Luego, este modelo fue empleado por Boyer, Verhaar, Roy, Biron \& Morin (2010), y los resultados mostraron que a partir del 2010 al 2099 habrá un aumento en el suministro de sedimento y extensión de las zonas de acumulación, el cual logrará modificar la extensión de los humedales de agua dulce en la desembocadura de los tributarios de Saint Lawrence.

Por otra parte, otro de los métodos usados para evaluar el efecto del cambio climatico sobre el transporte de sedimento en grandes ríos es el modelo de la Red Neuronal Artificial (RNA) (Zhu, Lu \& Zhou, 2008), el cual es un modelo empírico que tiene capacidad para simular sistemas complejos no lineales sin asumir ninguna prioridad sobre los procesos involucrados (ASCE, 2000), y recientemente han ganado popularidad en el campo de estudio de sedimentos (Zhao, Yang, Yang \& Huang, 2017; Yadav, Chatterjee, \& Equeenuddin, 2017).

Por otro lado, a los modelos empleados deben realizarse un análisis de la incertidumbre de las estimaciones para garantizar la calidad de la información estimada (Potemkina \& Potemkin, 2015). Fischer, Pietron, Bring, Thorslund \& Jarsjö (2017) utilizando el modelo unidireccional HEC-RAS y considerando las condiciones climáticas presentes y futuras, estimaron altas incertidumbres en las estimaciones (270-720 Mt año ${ }^{-1}$ ) debido a la insuficiente disponibilidad de datos medidos de cargas de sedimento y otros parámetros necesarios para el modelo de transporte de sedimento en el río Brahmaputra (Asia), los cuales deben analizarse para obtener estimaciones veraces. En el estudio realizado por Gómez et al. (2009), los autores concluyeron que se estimaron disminuciones en la descarga de sedimento suspendidos en el río Waipaoa (Nueva Zelanda) durante las variaciones del clima en el siglo XXI, pero con el análisis de los modelos empleados TUGS e HydroTend, los autores manifestaron que se pudieron presentar estimaciones no reales, ya que el modelo aunque responde bien a variaciones de precipitación y temperatura, es sensible a los cambios en la cobertura del suelo, que no fue tomada en cuenta en la investigación. Es por ello que debe considerarse el cambio uso del suelo, actividades humanas y el clima para evaluar la sensibilidad en las estimaciones de los modelos empleados de transporte de sedimentos, con el objeto de garantizar estimaciones fiables y holísticas.

\section{Influencia del cambio climático y el uso del suelo en el transporte de sedimentos}

Dado que tanto el cambio climático y el uso de suelo pueden interactuar en el tiempo modificando las tasas de transporte de sedimento en los ríos, se hace pertinente revisar esta temática. Wang et al. (2007) estudiaron las variaciones interanuales y estacionales de la carga de sedimento del río Amarillo (China) a partir de un conjunto de datos del periodo 1950-2005 registrado en cuatro estaciones distribuidas en el río. Dicho análisis permitió concluir que el río Amarillo ha experimentado disminuciones de las tasas de transporte de sedimento debido al cambio climático y a actividades humanas extensas. Zhao et al. (2015) inspeccionaron las influencias del cambio climático y las actividades humanas sobre los cambios temporales en la descarga de agua y la carga de sedimento en la cuenca del río Yangtze de 1953 al 2010 utilizando la prueba de Mann-Kendall y el método de onda de Morlet, y encontraron que el cambio climático (principalmente la precipitación) y las 
actividades humanas afectaron en conjunto la carga de sedimento en la cuenca del rio de estudio. Conclusiones similares se obtuvieron en el río Amarillo (China) en un estudio realizado por Yao \& Xu (2013) usando una metodología similar.

Gao, Geissen, Ritsema, Mu \& Wang (2013) aplicando los métodos de Petit y curvas de doble masa, reportan que las reducciones en el caudal y la descarga de sedimento fueron más atribuibles a la actividad humana que a la precipitación en la cuenca del río Wei (China); por lo que en ausencia de precipitaciones es importante buscar estrategias para controlar la descarga de sedimento en las cuencas hidrográficas. Estudio realizado por Jiang, Zhang \& Tang (2017) determinaron mediante la prueba no paramétrica de MannKendall, la prueba de Pettitt y la curva acumulativa, que el cambio climático desempeñó un papel importante en la reducción de la descarga de sedimento en las cabeceras de la cuenca de los ríos Amarillo y Yangtze.

Wei et al. (2016) analizaron las razones del cambio del caudal y la descarga de sedimento, así como la predicción de las tendencias futuras en el río Amarillo; aplicando el test no paramétrico de Mann-Kendall, el estimador de inclinación de Sen, el test de Pettitt y la transformada wavelet. Los resultados revelaron que las actividades humanas fueron las principales causas de la disminución del caudal y descarga de sedimento suspendido con reducciones de 49.7-87.8\%, mientras que el cambio climático en menor proporción oscilando entre 12.2-50.3\%. Liu et al. (2013), encontraron resultados similares, concluyendo que la disminución del transporte de sedimentos en diez grandes ríos de China que desembocan en el Océano Pacifico se le atribuyó a los efectos entre actividades humanas y cambio climático, pero este se basó en la determinación de un parámetro denominado Trend Ratio T". Por el contrario, en el estudio realizado por Restrepo \& Escobar (2016), los autores reportan que las tasas crecientes de transporte de sedimento fueron atribuibles principalmente a la erosión de la cuenca del río Magdalena (Colombia) por actividades forestales y no debe atribuirse únicamente al aumento de precipitación y cambio climático. Por lo que se hace importante analizar en conjunto el uso del suelo y el cambio climático en el transporte de sedimento.

Wang et al. (2011) construyeron una serie temporal de datos sobre las descargas anuales de agua y los flujos de sedimentos de cinco grandes ríos (del este y el sureste asiático) al Océano Pacífico occidental en un periodo de 1950-2008, los autores mencionan que el flujo sedimentario tuvo una tendencia decreciente y la variación a corto plazo (escala interanual) es dominada por oscilaciones climáticas como el Niño y La Niña, y a largo plazo (escala decadal y milenaria) está controlada por intervenciones humanas incluyendo embalses y cambios de uso del suelo que ocasionen erosión del suelo. Esto concuerda con lo reportado por Liu et al. (2014) en tres ríos chinos que descargan en el Océano Pacifico, realizando análisis y comparación de los datos actualizados del 2011 en las escalas estacional y anual; Wang et al. (2010), reportan que las influencias del cambio climático y actividades humanas han reducido la descarga de sedimento al mar en un $90 \%$ durante las últimas seis décadas en la desembocadura del rio Amarillo al océano.

Por otra parte, Potemkina \& Potemkin (2015) examinaron la sensibilidad del flujo de sedimentos al cambio climático y al impacto de la actividad humana y su interacción en la formación del transporte de sedimento en los principales ríos que desembocan en el Lago Baikal (Rusia) analizando datos del Servicio Federal de Hidrometeorología y 
Monitoreo Ambiental de Rusia. En los resultados obtenidos, se observó una tendencia de disminución en el transporte de sedimento (49-82\%), la cual se infirió que es causada tanto por la influencia antropogénica como por el cambio climático. Este resultado es similar al reportado por Yu et al. (2011), los autores mencionan que la evolución de la línea de costa y el cambio del área del delta del río Amarillo están siendo afectados directamente por la reducción significativa de la escorrentía y carga de sedimento, que están ligadas a actividades humanas relacionadas con la cuenca hidrográfica del río de estudio, así como con el cambio climático.

He et al. (2016) desarrollaron una técnica que podría ayudar a revelar las relaciones no lineales entre el clima, las actividades humanas, la escorrentía y la descarga de sedimentos en los ríos; en un estudio que se realizó en el Río Amarillo; basados en el Índice de Vegetación de Diferencia Normalizada (IVDN), en datos de descarga de sedimento, la escorrentía y los datos meteorológicos mensuales 1961-2010), en el análisis de la precipitación promedio mensual regional y la evapotranspiración potencial mediante el método Penman-Monteith, Los autores menciona que tanto el cambio climático como las actividades humanas redujeron la concentración de sedimento en el río, siendo la segunda la más impactante. En el estudio realizado por Tang et al. (2012), se menciona algo similar, es decir, que la reducción significativa de tasas de sedimento se debió a las variaciones de las precipitaciones y a actividades humanas locales; aunque en este el análisis realizado fue diferente, ya que realizaron estimaciones con la determinación de la curva de doble masa y modelos de regresión lineal en el río Mingjiang (China).

Por otro lado, Bussi et al. (2016) propusieron una nueva metodología basada en la extensión de la metodología de escenario neutro para explicar los efectos conjuntos del cambio climático y el cambio del uso de suelo. En este estudio usaron el modelo INCA para estimar tasas de transporte de sedimento en el río Támesis (Europa), proyectando el clima y planteando escenarios de cobertura del suelo; logrando concluir que esta metodología conlleva a resultados sólidos y que la precipitación es dominante en el transporte de sedimento.

Otras consideraciones en la modelación, es su aplicación para evaluar periodos de tiempo largos. Como por ejemplo: Wei, Balen, Verstraeten, Renssen \& Vandenberghe (2009) emplearon el modelo de distribución espacial de erosión del suelo y sedimentos (WATEM/SEDEM) y modelos climáticos ECBilt-CLIO-VECODE para evaluar la variación de sedimentos suspendidos en el pasado [ 4000-3000 A.P. (antes del presente) ] y en el futuro [ 1000-2000 D.P. (después del presente) ] y el siglo XXI, en la cuenca Meuse (Europa). Los resultados indicaron que, a escala de tiempo milenaria, casi todo el aumento de sedimento suspendido en la cuenca de estudio pudo atribuirse a los efectos del cambio antropogénico del uso de la tierra; sin embargo, a medida que aumenta la deforestación, la sensibilidad del sedimento suspendido atribuible al cambio climático también incrementa.

En contraste a los estudios mostrados anteriormente, es importante evaluar por separado los efectos del cambio climático y las actividades humanas sobre el transporte de sedimento, ya que las actividades humanas (como por ejemplo: el cultivo, la deforestación, la minería y la construcción de presas, etc.) que alteran la dinámica sedimentaria, confunden con el impacto del complejo cambio climático (Naik \& Jay, 2011), y por ende, en las incertidumbres de las estimaciones. En un estudio realizado por Naik \& Jay (2011) analizaron por separado las influencias humanas y climáticas en el ciclo hidrológico y el 
transporte de sedimento en el río Columbia (América del Norte) mediante un análisis de datos desde 1958. Los resultados obtenidos permitieron concluir que la mayor parte de la reducción en el transporte de sedimento en el interior de la sub-cuenca del río estudiado se relacionó con el sistema de presas.

Por otro lado, Houben, Hoffmann, Zimmermann \& Dikau (2006) propusieron una metodología para analizar las tasas de sedimento a partir de datos espacialmente distribuidos disponibles (mapas del suelo, mapas geológicos, DEM) en un estudio en el río Rin. Además, se esbozó un nuevo enfoque estadístico que utilizaron datos arqueológicos, proporcionando series temporales de impacto humano cuantificado (densidades de la población y las relaciones con el suelo y bosques abiertos). En este estudio, se concluyó principalmente que el trabajo metodológico presentado fue útil para evaluar la calidad de los datos disponibles, cuando estos se utilizan para la calcular las tasas de sedimento y para cuantificar el impacto humano; sin embargo, se sugiere que se requiere de un modelo refinado para mejorar la estimación del almacenamiento de sedimento.

Como alternativa para optimizar los métodos más comunes para separar las contribuciones climáticas y antropogénicas en la carga de sedimentos, Zhao et al. (2017) elaboraron una metodología poco usada, acoplando un modelo hidrológico mensual agrupado y un modelo de Red Neuronal Artificial (RNA) para diferenciar los impactos antropogénicos y climáticos sobre la carga de sedimentos en la cuenca del río Huangfuchuan. Finalmente, este tipo de análisis también es importante, ya que se están integrando nuevos métodos para estimar y entender un problema muy complicado como lo es el cambio climático, uso de suelo, y sus relaciones con el transporte de sedimentos.

\section{Conclusiones}

En este trabajo se logró revisar la literatura científica sobre los impactos del cambio climático y su incidencia sobre el transporte de sedimento en grandes ríos. Sin embargo, esta investigación permitió conocer la importancia de analizar el cambio climático de manera simultánea e integrada con el desarrollo de actividades humanas en cuencas hidrográficas (como por ejemplo: agricultura, construcción de presas, turismo, embalses, entre otras), ya que se han presentado casos de estudio en los cuales la afectación de las tasas de transporte de sedimento ha sido más influenciada por el uso de suelo (como por ejemplo: la construcción de presas), que por el cambio climático, que aunque este último es evidente, muchas veces su análisis individual no representa bien su grado de incidencia sobre el transporte de sedimento, ya que al no analizar las actividades antropogénicas, en especial en cuencas muy ocupadas y/o gestionadas con programas de conservación de agua y suelo, se correría el riesgo de tener errores muy altos en las estimaciones realizadas.

Adicionalmente, se identificó que el cambio climático y su afectación en las tasas de transporte de sedimentos en ríos al ser un tema de interés actual, requiere de nuevos enfoques y métodos que permitan mejorar su estimación e integración entre los mismos, como: Análisis SIG, modelos no tradicionales (como por ejemplo: RNA), análisis de isotopía, entre otros, los cuales también deben considerar el efecto antropogénico sobre la dinámica sedimentaria en ríos tanto en conjunto como de manera separada de las diferentes 
actividades humanas en las cuencas hidrográficas. Así mismo, se hace necesario que estos métodos sean robustos para que disminuyan su sensibilidad a los cambios de los parámetros que conllevan a errores con el fin de tener cálculos precisos y confiables que sirvan de base para que se tomen decisiones acertadas sobre la gestión de esta problemática desde un enfoque holístico e integral.

\section{Referencias bibliográficas}

ASCE. (2000). Artificial neural networks in hydrology. I: Preliminary concepts. Journal of Hydrologic Engineering, 5(2), 115-123

Asselman, N., Middelkoop, H. \& Dijk, P. (2004). The impact of changes in climate and land use on soil erosion, transport and deposition of suspended sediment in the River Rhine. Hydrol. Process, 17, 3225-3244.

Beltaos, S. \& Burrell, B. (2016). Transport of suspended sediment during the breakup of the ice cover, Saint John River, Canada. Cold Regions Science and Technology, $129,1-13$.

Beltaos, S. (2016). Extreme sediment pulses during ice breakup, Saint John River, Canada. Cold Regions Science and Technology, 128, 38-46.

Bogaart, P. \& Balen, R. (2000). Numerical modeling of the response of alluvial rivers to Quaternary climate change. Global and Planetary Change, 27, 147-163.

Bogen, J. (2009). The impact of environmental changes on the sediment loads of Norwegian rivers. Catena, 79, 251-256.

Boyer, C., Verhaar, P., Roy, A., Biron, P. \& Morin, J. (2010). Impacts of environmental changes on the hydrology and sedimentary processes at the confluence of St. Lawrence tributaries: potential effects on fluvial ecosystems. Hydrobiologia, 647, 163-183.

Bussi, G., Dadson, S., Prudhomme, C. \& Whitehead, P. (2016). Modelling the future impacts of climate and land-use change on suspended sediment transport in the River Thames (UK). Journal of Hydrology, 542, 357-372.

Bussi, G., Francés, F., Horel, E., López, J. \& Batalla, R. (2014). Modelling the impact of climate change on sediment yield in a highly erodible Mediterranean catchment. J Soils Sediments, 14, 1921-1937.

Darby, S., Dunn, F., Nicholls, R., Rahman, M. \& Riddy, L. (2015). A first look at the influence of anthropogenic climate change on the future delivery of fluvial sediment to the Ganges-Brahmaputra-Meghna delta. Environ. Sci.: Processes Impacts, 17, 1587-1600.

Fischer, S., Pietron, J., Bring, A., Thorslund, J. \& Jarsjö, J. (2017). Present to future sediment transport of the Brahmaputra River: reducing uncertainty in predictions and management. Reg Environ Change, 17, 515-526. 
Francés, F. \& Bussi, G. (2015). Análisis del impacto del cambio climático en el ciclo de sedimentos de la cuenca del río Ésera (España) mediante un modelo hidrológico distribuido. RIBAGUA - Revista Iberoamericana del Agua, 1, 14-25.

Fuentes, J. (2000). Iniciación a la meteorología y la climatología. Barcelona: Mundi- Presa.

Gao, P., Geissen, V., Ritsema, C., Mu, X. \& Wang, F. (2013). Impact of climate change and anthropogenic activities on stream flow and sediment discharge in the Wei River basin, China. Hydrol. Earth Syst. Sci, 17, 961-972.

Gómez, B., Cui, Y., Kettner, A., Peacock, D. \& Syvitski, J. (2009). Simulating changes to the sediment transport regime of the Waipaoa River, New Zealand, driven by climate change in the twenty-first century. Global and Planetary Change, 67, 153-166.

He, Y., Wang, F., Mu, X., Guo, L., Gao, P. \& Zhao, G. (2016). Human activity and climate variability impacts on sediment discharge and runoff in the Yellow River of China. Theor Appl Climatol, 129(1-2), 645-654.

Houben, P., Hoffmann, T., Zimmermann, A. \& Dikau, R. (2006). Land use and climatic impacts on the Rhine system (RheinLUCIFS): Quantifying sediment fluxes and human impact with available data. Catena, 66, 42-52.

IPCC (Intergovernmental Panel on Climate Change). (2007). Climate change: AR4 synthesis report. Recuperado de: https://www.ipcc.ch/site/assets/uploads/2018/02/ar4_ syr_full_report.pdf.

Jiang, C., Zhang, L. \& Tang, Z. (2017). Multi-temporal scale changes of streamflow and sediment dischargein the headwaters of Yellow River and Yangtze River on the TibetanPlateau, China. Ecological Engineering, 102, 240-254.

Kazimierski, L., Re, M. \& Menéndez, A. (2011). Influencia del cambio climático sobre la sedimentación en canales de navegación. En: Farías (Presidencia), Quinto simposio regional sobre hidráulica de ríos. Simposio llevado a cabo en la quinta edición del simposio regional sobre hidráulica de ríos, Santiago del Estero, Argentina.

Khan, M., Daityari, S. \& Chakrapani, G. (2016). Factors responsible for temporal and spatial variations in water and sediment discharge in Ramganga River, Ganga Basin, India. Environ Earth Sci, 75, 283.

Liu, C., Sui, J., He, Y. \& Hirshfield, F. (2013). Changes in runoff and sediment load from major Chinese rivers to the Pacific Ocean over the period 1955-2010. International Journal of Sediment Research, 28, 486-495.

Liu, F., Yang, Q., Chen, S., Luo, Z., Yuan, F. \& Wang, R. (2014). Temporal and spatial variability of sediment flux into the sea from the three largest rivers in China. Journal of Asian Earth Sciences, 87, 102-115.

Lu, X., Ran, L., Liu, S., Jiang, T., Zhang, S. \& Wang, J. (2013). Sediment loads response to climate change: A preliminary study of eight large Chinese rivers. International Journal of Sediment Research, 28, 0-14. 
Marrero, A., Tudurí, A., Pérez, L., Cuña, C., Muniz, P., Lopes, R., Michcelovitch, M., Alves, P., Pittauerová, D., Hanebuth, T. \& García, F. (2014). Cambios históricos en el aporte terrigeno de la cuenca del Río de la Plata sobre la plataforma interna uruguaya. Latin American Journal of Sedimentology and Basin Analysis, 21(2), 165-179.

Mei'e, R. (2015). Sediment discharge of the Yellow River, China: past, present and future-A synthesis. Acta Oceanol. Sin., 34(2), 1-8.

Mullan, D., Favis-Mortlock, D. \& Fealy, R. (2012). Addressing key limitations associated with modelling soil erosion under the impacts of future climate change. Agric For Meteorol, 156, 18-30.

Muñoz, E. \& Castillo, M. (2015). Streamflow and sediment load assessment from 1950 to 2006 in the Usumacinta and Grijalva Rivers (Southern Mexico) and the influence of ENSO. Catena, 127, 270-278.

Naik, P. \& Jay, D. (2011). Distinguishing human and climate influences on the Columbia River: Changes in mean flow and sediment transport. Journal of Hydrology, 404, 259-277.

Owens, P. \& Walling, D. (2002). Changes in sediment sources and floodplain deposition rates in the catchment of the River Tweed, Scotland, Over the last 100 years: The impact of climate and land use change. Earth Surf. Process. Landforms, 27, 403-423.

Potemkina, T. \& Potemkin, V. (2015). Sediment load of the main rivers of Lake Baikal in a changing environment (east Siberia, Russia). Quaternary International, 380381, 342-349.

Programa de las Naciones Unidas para el Medio Ambiente (PNUMA). (2005). Cambio climático. Manual de ciudadanía ambiental global. Ciudad de México: Programa de las Naciones Unidas para el Medio Ambiente.

Pruski, F. \& Nearing, M. (2002). Climate-induced changes in erosion during the 21st century for eight US locations. Water Resour Res, 38(12), 34-1-34-11.

Quynh, T., Garnier, J., Gilles, B., Sylvain, T. \& Minh, C. (2007). The changing flow regime and sediment load of the Red River, Viet Nam. Journal of Hydrology, 334, 199-214.

Re, M., Kazimierski, L. \& Menéndez, A. (2014). Sedimentación en canales de navegación en el contexto del cambio climático. Tecnología y Ciencias del Agua, 5(1), 5-21.

Restrepo, J. \& Escobar, H. (2016). Sediment load trends in the Magdalena River basin (1980-2010): Anthropogenic and climate-induced causes. Geomorphology, 302, 76-91.

Rocha, A. (1998). Introducción a la Hidráulica fluvial. Lima: Universidad Nacional de Ingeniería-Facultad de Ingeniería Civil.

Scherler, D., Bookhagen, B., Wulf, H., Preusser, F. \& Strecker, M. (2015). Increased late Pleistocene erosion rates during fluvial aggradation intheGarhwal Himalaya, northern India. Earth and Planetary Science Letters, 428, 255-266. 
Secretaría de Medio Ambiente y Recursos Naturales (SEMARNAT). (2009). Cambio climático. Ciencia, evidencia y acciones. Tlalpan: Secretaría de Medio Ambiente y Recursos Naturales.

Shrestha, B., Babel, M., Maskey, S., Griensven, A., Uhlenbrook, S., Green, A. \& Akkharath, I. (2013). Impact of climate change on sediment yield in the Mekong River basin: a case study of the Nam Ou basin, Lao PDR. Hydrol. Earth Syst. Sci., $17,1-20$.

Shrestha, B., Maskey, S., Babel, M., Griensven, A. \& Uhlenbrook, S. (2016). Sediment related impacts of climate change and reservoir development in the Lower Mekong River Basin: a case study of the Nam Ou Basin, Lao PDR, 149(1), 13-27.

Tang, Q., He, X., Bao, Y., Zhang, X., Guo, F. \& Zhu, H. (2012). Determining the relative contributions of climate change and multiple human activities to variations of sediment regime in the Minjiang River, China. Hydrol. Process, 27(25), 3547-3559.

Verhaar, P., Biron, P., Ferguson, R. \& Hoey, T. (2008). A modified morphodynamic model for investigating the response of rivers to short-term climate change. Geomorphology, 101, 674-682.

Wang, H., Bi, N., Saito, Y., Wang, Y., Sun, X., Zhang, J. \& Yang, Z. (2010). Recent changes in sediment delivery by the Huanghe (Yellow River) to the sea: Causes and environmental implications in its estuary. Journal of Hydrology, 391, 302-313.

Wang, H., Saito, Y., Zhang, Y., Bi, N., Sun, X. \& Yang, Z. (2011). Recent changes of sediment flux to the western Pacific Ocean from major rivers in East and Southeast Asia. Earth-Science Reviews, 108, 80-100.

Wang, H., Yang, Z., Saito, Y., Liu, J., Sun, X. \& Wang, Y. (2007). Stepwise decreases of the Huanghe (Yellow River) sediment load (1950-2005): Impacts of climate change and human activities. Global and Planetary Change, 57, 331-354.

Wei, J., He, X. \& Bao, Y. (2011). Anthropogenic impacts on suspended sediment load in the Upper Yangtze river. Reg Environ Change, 11, 857-868.

Wei, Y., Jiao, J., Zhao, G., Zhao, H., He, Z. \& Mu, X. (2016). Spatial-temporal variation and periodic change in streamflow and suspended sediment discharge along themainstreamof the YellowRiver during 1950-2013. Catena, 140, 105-115.

Yadav, A., Chatterjee, S. \& Equeenuddin, S. (2017). Prediction of suspended sediment yield by artificial neural network and traditional mathematical model in Mahanadi river basin, India. Sustainable Water Resources Management, 4(4), 745-759.

Yao, W. \& Xu, J. (2013). Impact of human activity and climate change on suspended sediment load: the upper Yellow River, China. Environ Earth Sci, 70, 1389-1403.

Yu, J., Fu, Y., Li, Y., Han, G., Wang, Y., Zhou, D., Sun, W., Gao, Y. \& Meixner, F. (2011). Effects of water discharge and sediment load on evolution of modern Yellow River Delta, China, over the period from 1976 to 2009. Biogeosciences, 8, 2427-2435. 
Zhao, H., Yang, S., Yang, B. \& Huang, Y. (2017). Quantifying anthropogenic and climatic impacts on sediment load in the sediment-rich region of the Chinese Loess Plateau by coupling a hydrological model and ANN. Stoch Environ Res Risk Assess, 31(8), 2057-2073.

Zhao, Y., Zou, X., Gao, J., Xu, X., Wang, C., Tang, D., Wang, T. \& Wu, X. (2015). Quantifying the anthropogenic and climatic contributions to changes in water discharge and sediment load into the sea: A case study of the Yangtze River, China. Science of the Total Environment, 536, 803-812.

Zhu, Y., Lu, X. \& Zhou, Y. (2008). Sediment flux sensitivity to climate change: A case study in the Longchuanjiang catchment of the upper Yangtze River, China. Global and Planetary Change, 60, 429-442. 